\title{
METAL SURFACE DIPOLE MOMENT AND WORK FUNCTION IN THE LINEAR POTENTIAL MODEL
}

\author{
A.E. MOHammed \\ Mathematics Department, Faculty of Science, Ain Shams University, Egypt \\ A.Y. Ghaly, and O.M. Frege \\ Mathematics Department, Faculty of Education, Ain Shams University, Egypt \\ (Received October 15, 1990)
}

\begin{abstract}
Jellium metal surface properties such as the surface dipole barrier and work function are obtained in the linear potential approximation to the effective potential at the surface. The metal surface position and field strength are determined respectively by the requirement of overall charge neutrality and the constraint set on the electrostatic potential by the Budd-Vannimenus theorem. The calculations are primarily analytic and these properties are given in terms of universal functions of the field strength. The results obtained employing the Ceperly-Alder expression for the correlation energy closely approximate those of Lang and Kohn.
\end{abstract}

PACS numbers: $73.30 .+\mathrm{y}$

\section{Introduction}

In this paper we present the results of a model potential calculation of jellium metal [1] surface properties. The principal advantage of such model calculation [2, 3 ] is the elimination of the requirement of a numerical solution of Schrödinger equation for particles moving in a self consistently obtained effective potential which is intrinsic to other more complex formalisms $[4,5]$. Together with the application of certain theoretical constraint, which help to define the model effective potential, it is then possible to determine various properties of interest, such as a work function and surface energies. Examples of typical constraints applicable to model metal surface calculations are the requirement of self consistency of the surface dipole 
barrier [2], the condition on the electrostatic potential at the metal surface set by the Budd-Vannimenus theorem [6] (BVT), and the Rayleigh-Ritz [7] energy minimum criterion in addition to the charge neutrality condition [8]. The choice and number of these constraints to be satisfied would, of course, depend upon the complexity of the model potential empoyed.

We consider here the linear potential model $[9,10]$ approximation to the effective potential at a metal surface (Fig. 1). For a given value of the field strength, the metal surface position is fixed by requiring overall charge neutrality. The field strength may then be determined by application of the BVT. The choice of BVT criterion as the constraint is governed by the fact that its application in the previous work [3] consistently lead to good results for the work function. In Sec. 2 we give a discussion of the calculations and definitions of properties, and theorems employed. Finally we compare our results obtained by employing Ceperly-Alder approximation for correlation with those of self consistent calculations of Lang and Kohn [5] and also with those of employing Wigner approximation for correlation [11].

\section{Calculation of metal surface dipole barrier and work function}

In this model calculation the effective potential $V_{\text {eff }}(x)$ at a metal surface (Fig. 1) is assumed to be:

$$
V_{\mathrm{efr}}(x)=F x \theta(x),
$$

where $F$ is the field strength defined in terms of the slope parameter $x_{F}$ as* $F=k_{\mathrm{F}}^{2} / 2 x_{\mathrm{F}}, k_{\mathrm{F}}^{2} / 2$ is the Fermi energy and $\theta(x)$ is the step function.

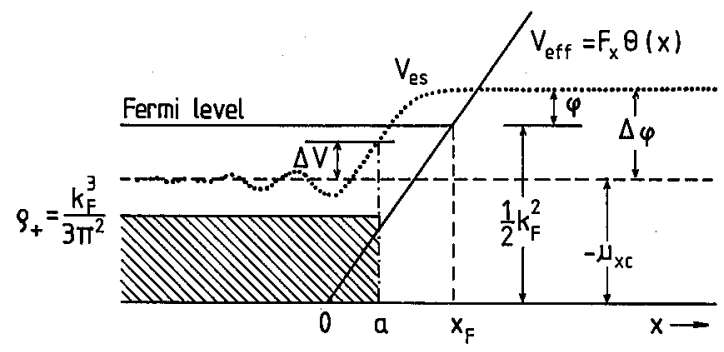

Fig. 1. Schematic representation of the linear potential model of a metal surface indicating all relevant energies. The hatched region represents the uniform positive background beginning at the metal surface.

The electronic wave function $\psi_{k}(x)$ for $x \leq 0$ is given as

$$
\psi_{k}(x)=-\sqrt{\frac{2}{L}} \sin [k x+\delta(k)]
$$

\footnotetext{
*Atomic units are used $|e|=h=m=1$. The unit of energy is $27.21 \mathrm{eV}$.
} 
in the region $x \geq 0$ the Schrödinger equation is the Airy differential equation $[12,13]$ :

$$
\frac{\mathrm{d}^{2} \psi_{k}}{\mathrm{~d} \xi^{2}}-\xi \psi_{k}=0
$$

whose solution is

$$
\psi_{k}(\xi)=C_{k} A i(\xi)
$$

where $A i(\xi)$ is the Airy function, $C_{k}$ - normalization factor, $\xi=(x-E / F)(2 F)^{1 / 3}$, and $E$ is the energy. The factor $C_{k}$ and the phase shift $\delta(k)$ are determined by the requirement of the continuity of the wave function and its logarithmic derivative at the origin. Thus

$$
C_{k}=-\sqrt{\frac{2}{L}} \sin \delta\left(k_{\mathrm{F}}, x_{\mathrm{F}}\right)\left[A i\left(-\xi_{0}\right)\right]^{-1}
$$

and

$$
\cot \delta\left(k_{\mathrm{F}}, x_{\mathrm{F}}\right)=\frac{1}{\sqrt{\xi_{0}}} \frac{A i^{\prime}\left(-\xi_{0}\right)}{A i\left(-\xi_{0}\right)}
$$

where $\xi_{0}=\left(k^{2} / k_{\mathrm{F}}\right)\left(k_{\mathrm{F}} x_{\mathrm{F}}\right)^{2 / 3}$, and $A i^{\prime}(\xi)$ is the derivative of the Airy function. The fundamental quantities from which all other surface properties may be obtained are the electronic and total charge densities defined as:

$$
\rho_{\mathrm{e}}(x)=\frac{L}{2 \pi^{2}} \int_{0}^{k_{\mathrm{F}}}\left(k_{\mathrm{F}}^{2}-k^{2}\right)\left|\psi_{k}(x)\right|^{2} \mathrm{~d} k
$$

and

$$
\rho_{\mathrm{t}}(x)=\rho_{\mathrm{e}}(x)-\frac{k_{\mathrm{F}}^{2}}{3 \pi^{2}} \theta(-x+a),
$$

respectively. Implicit in the definition of the total charge density is the assumption that the positive ions of the metal are smeared out and replaced by a uniform charge background of density $\rho_{+}=k_{\mathrm{F}}^{3} / 3 \pi^{2}$ ending abruptly at the metal surface position at $x=a$.

The surface dipole barrier contribution to the work function is given by the expression:

$$
\Delta \varphi=4 \pi \int_{-\infty}^{\infty} x \rho_{\mathrm{t}}(x) \mathrm{d} x .
$$

The electrostatic potential $V_{\mathrm{es}}(x)$ required for the application of BVT is obtained by solution of Poisson's equation:

$$
\frac{\mathrm{d}^{2} V_{\mathrm{es}}}{\mathrm{d} x^{2}}=-4 \pi \rho_{\mathrm{t}}(x)
$$

with the boundary conditions $V_{\mathrm{es}}(-\infty)=V_{\mathrm{es}}^{\prime}(-\infty)=0$. Applying the charge neutrality condition, the electrostatic potential may thus be written as:

$$
V_{\text {es }}(x)=\Delta \varphi-4 \pi \int_{+\infty}^{x} \mathrm{~d} x^{\prime} \int_{+\infty}^{x^{\prime}} \mathrm{d} x^{\prime \prime} \rho_{\mathrm{t}}\left(x^{\prime \prime}\right) .
$$


In this model the two variable parameters are the metal surface position $a$ and the field strength $F$. For a given $F$ the metal surface position may be determined either by charge neutrality condition:

$$
\int_{-\infty}^{\infty} \rho_{\mathrm{t}}(x) \mathrm{d} x=0
$$

or by application of the phase-shift rule of Sugiyama [15], according to which

$$
a=-\frac{3 \pi}{8 k_{\mathrm{F}}}-\frac{3}{k_{\mathrm{F}}^{3}} \int_{0}^{k_{\mathrm{F}}} k \delta(k) \mathrm{d} k .
$$

The field strength, or equivalently, the slope parameter $x_{\mathrm{F}}$ is adjusted so as to satisfy the requirement set on the electrostatic potential by the BVT such that

$$
\Delta V=V_{\mathrm{es}}(a)-V_{\mathrm{es}}(-\infty)=\rho_{\mathrm{e}} \frac{\mathrm{d} \varepsilon_{\mathrm{t}}}{\mathrm{d} \rho_{\mathrm{e}}}
$$

where $\varepsilon_{\mathfrak{t}^{t}}$ is the sum of the kinetic, exchange and correlation energies per partial for a uniform electron gas.

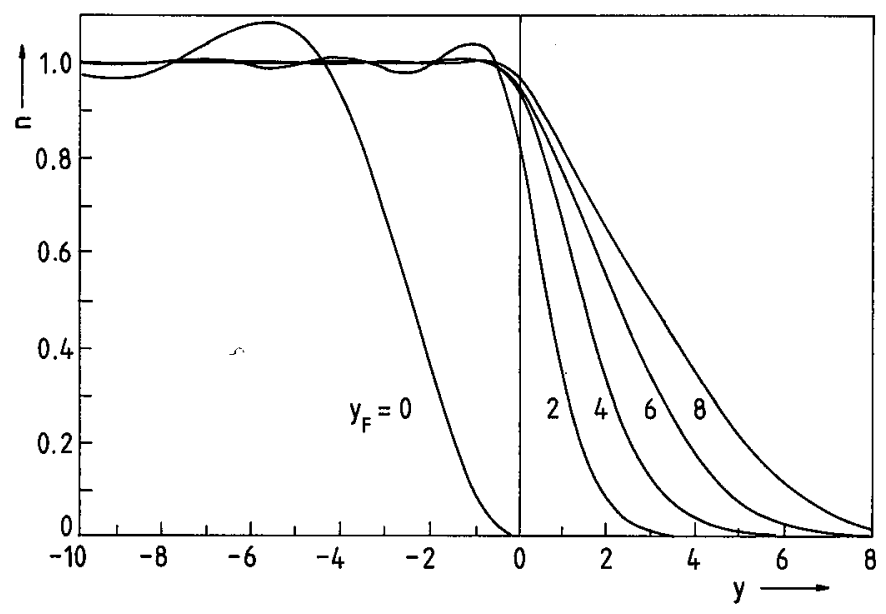

Fig. 2. Variation of the electronic charge density $n(y)$ as a function of the distance from the origin $y$, for the linear potential model for different values of the slope parameter: $y_{\mathrm{F}}=0.0, y_{\mathrm{F}}=2.0, \quad y_{\mathrm{F}}=4.0, y_{\mathrm{F}}=6.0$ and $y_{\mathrm{F}}=8.0$.

With the transformation $y=k_{\mathrm{F}} x$ and $q=k / k_{\mathrm{F}}$, such that the metal surface position is now defined to be at $y_{a}=k_{\mathrm{F}} a$, we have shown that the quantities $y_{a}$, $\rho_{\mathrm{e}} / k_{\mathrm{F}}^{3}, \Delta \varphi / l_{\mathrm{F}}$ and $V_{\mathrm{es}} / k_{\mathrm{F}}$ are all universal functions of the slope parameter $y_{\mathrm{F}}=$ $k_{\mathrm{F}} x_{\mathrm{F}}$. Furthermore, all the spatial integrals in the expressions for the metal surface position, the surface dipole barrier and the electrostatic potential can be done analytically. Thus together with the electronic charge density, the determination of these properties reduces to a simple numerical calculation of $k$-space integrals 
from 0 to 1 . The explicit expressions employed in the present calculations are given in the Appendix. Plots of the universal functions $n(y)=\rho_{\mathrm{e}} / \bar{\rho}, y_{a}$ and $\Delta \varphi / k_{\mathrm{F}}$ are given in Figs. 2-4. The metal surface position and surface dipole barrier are then

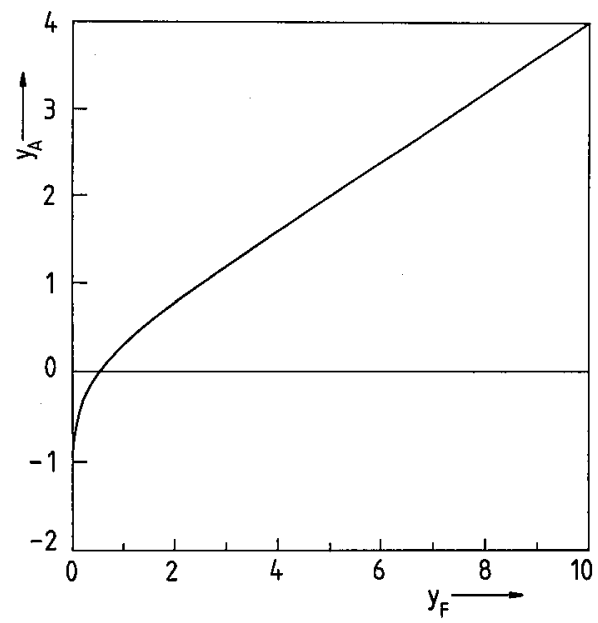

Fig. 3. Plot of the universal function for the metal surface position $y_{a}$ as a function of the slope parameter $y_{\mathrm{F}}$.

easily determined for a specific metal (defined by its Fermi momentum $k_{\mathrm{F}}=1 / \alpha r_{\mathrm{s}}$, $\left.\alpha^{-1}=(9 \pi / 4)^{1 / 3}\right)$ by adjusting the slope parameter $x_{\mathrm{F}}$ until it satisfies the BVT.

The work function of the metal $\varphi$ is then given as

$$
\varphi=\Delta \varphi-\frac{1}{2} k_{\mathrm{F}}^{2}-\mu_{\mathrm{xc}},
$$

where $\mu_{\mathrm{xc}}$ is the exchange and correlation part of the chemical potential of a uniform electron gas defined as:

$$
\mu_{\mathrm{xc}}=\frac{\mathrm{d}\left(\rho_{\mathrm{e}} \varepsilon_{\mathrm{xc}}\right)}{\mathrm{d} \rho_{\mathrm{e}}} .
$$

The exchange energy [15] per particle for the uniform electron gas is $\varepsilon_{\mathrm{x}}=$ $-0.458 / r_{s}$.

For the correlation energy per particle valid at metallic densities we employ the correlation function per particle of Wigner [16] and Ceperly-Alder [17, 18]:

$$
\varepsilon_{\mathrm{c}}^{\mathrm{W}}=\frac{-0.44}{\left(r_{s}+7.8\right)}
$$

and

$$
\varepsilon_{\mathrm{c}}^{\mathrm{CA}}=\frac{\gamma}{1+\beta_{1} \sqrt{r_{s}}+\beta_{2} r_{s}}
$$

where $\gamma=-0.1423, \beta_{1}=1.0529$ and $\beta_{2}=0.3334$. 


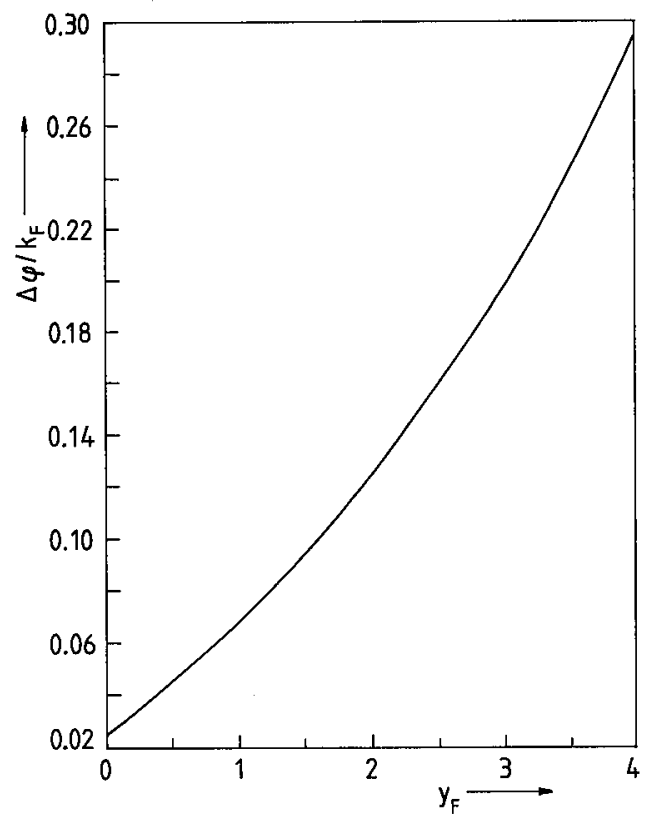

Fig. 4. Plot of the universal function $\Delta \varphi / k_{F}$, where $\Delta \varphi$ is the surface dipole barrier as a function of the slope parameter $y_{F}$.

The results for the surface dipole barrier and work function within the linear potential model employing these different correlations are presented in Sec. 3.

\section{Results}

In Table I we present the results for the surface dipole barrier and work function for the two different correlation functions given by Wigner (W) and Ceperly-Alder (CA). For $r_{s}=2 \div 5$ both the BVT and charge neutrality are exactly satisfied using Ceperly-Alder correlation formula, however for Wigner [12] formula these conditions are satisfied for $r_{s}=2 \div 4$. Whereas for $r_{s} \geq 5.5$, using CA formula, there is no choice of parameters in the linear potential model satisfying both these requirements. Thus for $r_{s} \geq 5.5$ the present work satisfies the BVT as closely as possible in the limit of this model, i.e. by a potential of infinite slope and by the charge neutrality condition.

A comparison of Ceperly-Alder (CA) results with those of Lang and Kohn (LK) indicate that the model reproduces the large dipole moments requried for high-density metals, differing the value of $\Delta \varphi$ by $0.08,0.03,0.01$ and $0.003 \mathrm{eV}$ for $r_{s}=2.0,2.5,3.0$ and 3.5 , respectively. The reason for the agreement of these results is due to adjusting $\Delta V$ so as to satisfy the BVT, one has already cbtained approximately $40 \%$ of the dipole barrier. The remaining contribution to $\Delta \varphi$ from charge outside the metal is determined accurately since the model permits a large electronic spillover. In addition, satisfaction of the BVT also leads to very accurate 
electronic densities in this range. For $r_{s}=4.0,4.5$, and 5.0 the model becomes progressively more reflecting as the potential rises more steeply leading to underestimates for the dipole barrier. Also $\Delta V$ in this range comprises only about $22 \%$ of LK value. For $r_{s} \geq 5.5$, for which the contribution of the dipole barrier to the work function is small, the results for $\Delta \varphi$ are within $0.22 \mathrm{eV}$ of those obtained by LK. In this range, the LK results also do not satisfy the BVT, although their results more closely satisfy this condition than do the results of the infinite barrier model [2]. Thus, over the entire metallic range the results of this model calculation for the dipole barrier and work function are within $0.32 \mathrm{eV}$ of those due to LK. The use of the CA correlation function leads to results for surface dipole barrier $\Delta \varphi$ and work function $\varphi$ which are more closely approximate to those of LK than the ones obtained employing the $W$ function.

TABLE I

Results for the surface dipole barrier $\Delta \varphi$ and work function $\varphi$ using the correlation function due to Wigner (W), Lang and Kohn (LK), and Ceperley-Alder (CA).

\begin{tabular}{|c|c|c|c|c|c|c|c|c|c|c|c|c|}
\hline \multirow[t]{2}{*}{$\overline{R_{S}}$} & \multicolumn{2}{|c|}{$y_{F}$} & \multicolumn{2}{|c|}{$y_{\mathrm{a}}$} & \multicolumn{3}{|c|}{$\Delta \varphi$} & \multicolumn{3}{|c|}{$\varphi$} & \multirow{2}{*}{$\begin{array}{c}A^{a^{a}} \\
\left(e^{2}\right)\end{array}$} & \multirow{2}{*}{$\begin{array}{c}\mathbf{B}^{\mathbf{d}} \\
(\mathrm{eV})\end{array}$} \\
\hline & W & CA & W & $\mathrm{CA}$ & W & CA & LK & W & $\mathrm{CA}$ & LK & & \\
\hline 2.0 & 3.760 & 3.650 & 1.487 & 1.444 & 7.02 & 6.723 & 6.80 & 4.11 & 3.920 & 3.89 & 0.22 & 0.030 \\
\hline 2.5 & 3.807 & 2.850 & 1.107 & 1.127 & 3.78 & 3.863 & 3.83 & 3.67 & 3.775 & 3.72 & 0.05 & 0.055 \\
\hline 3.0 & 1.967 & 2.155 & 0.763 & 0.843 & 2.13 & 2.338 & 2.32 & 3.31 & 3.490 & 3.50 & 0.19 & 0.010 \\
\hline 3.5 & 1.214 & 1.520 & 0.410 & 0.561 & 1.18 & 1.426 & 1.43 & 3.01 & 3.184 & 3.26 & 0.25 & 0.077 \\
\hline 4.0 & 0.507 & 0.680 & -0.037 & 0.093 & 0.59 & 0.691 & 0.91 & 2.47 & 2.742 & 3.06 & 0.32 & 0.318 \\
\hline 4.5 & 0.000 & 0.350 & -1.178 & -0.180 & 0.27 & 0.443 & 0.56 & 2.58 & 2.630 & 2.87 & 0.29 & 0.240 \\
\hline 5.0 & 0.000 & 0.250 & -1.178 & -0.292 & 0.25 & 0.354 & 0.35 & 2.63 & 2.590 & 2.73 & 0.10 & 0.140 \\
\hline 5.5 & 0.000 & 0.000 & -1.178 & -0.178 & 0.22 & 0.234 & 0.16 & 2.60 & 2.459 & 2.54 & 0.06 & 0.081 \\
\hline 6.0 & 0.000 & 0.000 & -1.178 & -0.178 & 0.20 & 0.205 & 0.04 & 2.57 & 2.413 & 2.41 & 0.16 & 0.003 \\
\hline
\end{tabular}

The $\mathrm{CA}$ and $\mathrm{W}$ results for $\Delta \varphi$ are equal within $0.3 \mathrm{eV}$. On the other hand the $\mathrm{CA}$ and $\mathrm{W}$ values for $\varphi$ differ by a maximum of $0.19 \mathrm{eV}$ for a given $r_{s}$.

\section{Conclusion}

We obtain jellium metal surface properties such as the surface dipole barrier and work function in the linear potential approximation to the effective potential at the surface. We determine the metal surface position and field strength respectively by the requirement of overall charge neutrality and the constraint set on the electrostatic potential by the BV theorem. The calculations are primarily analytic and these properties, are given in terms of universal functions of the field strength. The results obtained employing the Ceperly-Alder expression for correlation energy closely approximate those of Lang and Kohn.

We observe, in conclusion, that the linear potential model together with the constraint of the sum rule due to Budd and Vannimenus leads to results for all surface properties comparable to those obtained by Lang and Kohn for jellium metal. The fact that the effective potential does not become constant but increases indefinitely is unimportant, since the effective potential is in substantial error only in the region far from the metal surface where the electron density has exponentially decayed to a small fraction of its value at the surface. The majority of the calculations, as shown in the Appendix, is primarily analytic, and the universal curves permit a direct determination of surface properties on application of any theoretical constraint. 


\section{Appendix}

With the transformation $y=k_{\mathrm{F}} x, k / k_{\mathrm{F}}=q$, the slope parameter is $y_{\mathrm{F}}=$ $k_{\mathrm{F}} x_{\mathrm{F}}$, the metal surface is at $y_{a}=k_{\mathrm{F}} a$, and the variable $\xi$ is

$$
\xi=\left(y-q^{2} y_{\mathrm{F}}\right) y_{\mathrm{F}}^{-1 / 3} .
$$

We present below expression for the phase shift, electronic density, metal surface position, surface dipole barrier, and the electrostatic potential in terms of the universal function of the slope parameter $y_{F}$.

\section{Phase shift}

With the definition

$$
k\left(q, y_{\mathrm{F}}\right)=y_{\mathrm{F}}^{1 / 3} \frac{A i\left(-q^{2} y_{\mathrm{F}}^{2 / 3}\right)}{A i^{\prime}\left(-q^{2} y_{\mathrm{F}}^{2 / 3}\right)}
$$

such that

$$
k(0)=y_{\mathrm{F}}^{1 / 3} \frac{A i(0)}{A i^{\prime}(0)}=-1.37172 y_{\mathrm{F}}^{1 / 3},
$$

we have

$$
\delta\left(q, y_{\mathrm{F}}\right)=\cot ^{-1}\left[\frac{1}{q k\left(q, y_{\mathrm{F}}\right)}\right]
$$

\section{Electronic density}

For $y \leq 0$

$$
\frac{\rho_{\mathrm{e}}(y)}{\bar{\rho}_{\mathrm{e}}}=1-\frac{3}{2} \int_{0}^{1} \mathrm{~d} q\left(1-q^{2}\right) \cos 2\left[q y+\delta\left(q, y_{\mathrm{F}}\right)\right],
$$

for $y \geq 0$

$$
\frac{\rho_{\mathrm{e}}(y)}{\bar{\rho}_{\mathrm{e}}}=3 \int_{0}^{1} \mathrm{~d} q\left(1-q^{2}\right) \sin ^{2} \delta\left(q, y_{\mathrm{F}}\right) \frac{A i^{2}\left[\left(y-q^{2} y_{\mathrm{F}}\right) y_{\mathrm{F}}^{-1 / 3}\right]}{A i^{2}\left(1-q^{2} y_{\mathrm{F}}^{2 / 3}\right)} .
$$

The universal functions for the remaining properties were obtained using the following integral expressions:

$$
\begin{aligned}
& \int A i^{2}(\xi) \mathrm{d} \xi=\xi A i^{2}(\xi)-A{i^{\prime}}^{2}(\xi) \\
& \int \xi A i^{2}(\xi) \mathrm{d} \xi=\frac{1}{3}\left[\xi^{2} A i^{2}(\xi)-\xi A{i^{\prime}}^{2}(\xi)+A i(\xi) A i^{\prime}(\xi)\right] \\
& \int A{i^{\prime}}^{2}(\xi) \mathrm{d} \xi=\frac{1}{3}\left[-\xi^{2} A i^{2}(\xi)+\xi A{i^{\prime}}^{2}(\xi)+2 A i(\xi) A i^{\prime}(\xi)\right]
\end{aligned}
$$




\section{Metal surface position} pression:

Application of the charge neutrality condition of Eq. (12) leads to the ex-

$$
y_{a}=k_{\mathrm{F}} a=-\frac{3 \pi}{8}+\frac{2}{5} y_{\mathrm{F}}-\frac{3}{2} \int_{0}^{1} \mathrm{~d} q\left(1-q^{2}\right) \frac{\sin 2 \delta\left(q, y_{\mathrm{F}}\right)}{2 q} .
$$

An alternate expression is obtained from the Sugiyama phase-shift rule of Eq. (13):

$$
y_{a}=-\frac{3 \pi}{8}-3 \int_{0}^{1} \mathrm{~d} q q \delta\left(q, y_{\mathrm{F}}\right) .
$$

\section{Surface dipole barrier}

$$
\frac{\Delta \varphi}{k_{\mathbf{F}}}=\frac{4}{3 \pi}\left[J\left(y_{\mathrm{F}}\right)+k\left(y_{\mathrm{F}}\right)\right],
$$

where

$$
J\left(y_{\mathrm{F}}\right)=\frac{3}{4}\left(1-\frac{2}{3} y_{a}^{2}+\frac{\pi}{2} k(0)+\int_{0}^{1} \mathrm{~d} q \frac{\left(1-q^{2}\right) k^{2}}{1+k^{2} q^{2}}\right.
$$

and

$$
k\left(y_{\mathrm{F}}\right)=\frac{4}{35} y_{\mathrm{F}}^{2}-\frac{y_{\mathrm{F}}}{2} \int_{0}^{1} \mathrm{~d} q\left(1-q^{2}\right) q \sin 2 \delta\left(q, y_{\mathrm{F}}\right) .
$$

Electrostatic potential

For $y \leq 0$

$$
\begin{aligned}
& \frac{V_{\mathrm{es}}(y)}{k_{\mathrm{F}}}=\frac{4}{3 \pi}\left(J\left(y_{\mathrm{F}}\right)+\frac{3 \pi}{8} y+\frac{y_{a}^{2}}{2} \theta\left(y_{a}-y\right)+\frac{1}{2} y\left(2 y_{a}-y\right) \theta\left(y-y_{a}\right)\right) \\
& +\frac{3}{4} \int_{0}^{1} \mathrm{~d} q\left(1-q^{2}\right)\left(\frac{\cos 2 \delta\left(q, y_{\mathrm{F}}\right) \sin ^{2} q y}{q^{2}}+\frac{\sin 2 \delta\left(q, y_{\mathrm{F}}\right) \sin 2 q y}{2 q^{2}}\right) .
\end{aligned}
$$

For $y \geq 0$

$$
\begin{aligned}
& \frac{V_{\mathrm{es}}(y)}{k_{\mathrm{F}}}=\frac{\Delta \varphi}{k_{\mathrm{F}}}+\frac{2}{3 \pi}\left(y-y_{a}\right)^{2} \theta\left(y_{a}-y\right)-\frac{4 y_{\mathrm{F}}^{2 / 3}}{3 \pi} \int_{0}^{1} \mathrm{~d} q\left(1-q^{2}\right) \\
& \times \frac{\sin ^{2} \delta\left(q, y_{\mathrm{F}}\right)}{A i^{2}\left(-q^{2} y_{\mathrm{F}}^{2 / 3}\right)}\left[2 \xi^{2} A i^{2}(\xi)-2 \xi A i^{\prime 2}(\xi)-A i(\xi) A i^{\prime}(\xi)\right] .
\end{aligned}
$$




\section{References}

[1] N.D. Lang, in Solid State Physics, Advanced in Research and Application Eds. H. Ehrenreich, F. Seutz, D. Turnbull, Vol. 28, Academic Press, New York 1973, p. 243.

[2] V.Sahni, J.B. Krieger, J. Grunebaum, Phys. Rev. B 12, 3503 (1975);

V. Sahni, J. Grunebaum, Phys. Rev. B 15, 1929 (1977).

[3] S.S. Abdel Wahed, A.E. Mohammed, I.I. Hanna, Ain Shams Sc. Bull. 28A, 3 (1990).

[4] W. Kohn, L.J. Sham, Phys. Rev. A 140, 1133 (1965).

[5] W.D. Lang, W. Kohn, Phys. Rev. B 1, 4555 (1970); 3, 1215 (1971).

[6] H.F. Budd, J. Vannimenus, Phys. Rev. Lett. 31, 1218 (1973); Erratum 31, 1430 (1973).

[7] B.L. Miiseowitsch, Variational Principles, Interscience, New York 1966, p. 153.

[8] J. Bardeen, Phys. Rev. 49, 653 (1936).

[9] W.J. Swiatecki, Proc. Phys. Soc. Lond. A 64, 226 (1951).

[10] A. Sugiyama, J. Phys. Soc. Jpn. 15, 965 (1960).

[11] V. Sahni, J.B. Krieger, J. Grunebaum, Phys. Rev. B 15, 1941 (1977).

[12] L.D. Landau, E.M. Lifshitz, Quantum Mechanics, Pergamon, New York 1965, p. 71 .

[13] M. Abramowitz, I.A. Stegun, Handbook of Mathematical Functions, Dorer, New York 1965, p. 446.

[14] A. Sugiyama, J. Phys. Soc. Jpn. 15, 965 (1960).

[15] S. Raimes, Wave Mechanics of Electrons in Metals, North-Holland, Amsterdam 1961, p. 177

[16] D. Pines, Elementary Excitations in Solids, Benjamin, New York 1963, p. 94.

[17] J.P. Perdew, A. Zunger, Phys. Rev. B 23, 5048 (1981).

[18] D.M. Ceperley, B.J. Alder, Phys. Rev. Lett. 45, 566 (1980). 